\title{
Chemotactic Responses by Motile Bacteria
}

\author{
By F. W. K. SEYMOUR AND R. N. DOETSCH \\ Department of Microbiology, University of Maryland, \\ College Park, Maryland 20742, U.S.A.
}

(Received I9 March 1973: revised I4 May 1973)

\begin{abstract}
SUMMA RY
Chemotactic behaviour of ten species of motile bacteria from nine different genera toward over I 30 compounds was examined using a flat-glass capillary tube technique and microscopical observations on bacteria both individually and in mass. Negative chemotactic behaviour was uniformly exhibited against acidic $(\mathrm{pH}$ $\mathrm{I} \cdot 0$ to $3 \cdot 0$ ) and basic ( $\mathrm{pH}$ I0.0 to $\mathrm{I} 2 \cdot 0$ ) stimuli. Benzene, chloroform, acetone and ethanol, whilst not extensively interfering with translational movement, uncoupled tactic responses to gradients of acids or bases. Positive chemotactic behaviour towards a given carbohydrate or amino acid was a relatively constant though rather rare event, governed to some extent by the laboratory conditions imposed. The biological role of negative chemotaxis appears to be one of survival, whereas positive responses may be, in some cases, incidental or useless.
\end{abstract}

\section{INTRODUCTION}

Considerable interest in unicellular motile behaviour has recently been evinced in the hope of understanding the nature of the sensory transduction processes in these forms, e.g. leukocytes (Gamow, Bottger \& Barnes, I97I), photosynthetic bacteria (Clayton, 1958, 1964), Euplotes and Paramecium (Naitoh \& Eckert, I969a, b), and myxamoebae (Konijn et al. 1969). Studies on tactic responses of bacteria might, in fact, provide information basic to all sensory phenomena (Adler, r966a, 1969; Smith \& Doetsch, I969). Theories concerning observed behaviour of unicells accordingly abound (Adler, I969; Doetsch \& Hageage, I968; Hazelbauer \& Adler, I97I ; Doetsch, I971, I972; Berg \& Brown, 1972; Macnab \& Koshland, I972; Eckert, 1972). Response specificity (Adler, I969; Mesibov \& Adler, I972), the apparent location of binding proteins in the outer membranes (Kalckar, 197I), and the possible mode of sensing of concentration ratios (Dahlquist, Lovely \& Koshland, I972; Macnab \& Koshland, 1972), suggest analogies between positive bacterial chemotactic responses and sensory receptor processes in other, more highly developed, organisms.

Most of the work on positive chemotaxis in bacteria has been done using only a few strains of Escherichia coli or Salmonella typhimurium, and if generalizations concerning this phenomenon are to be made, a much wider range of bacteria needs to be examined. Accordingly, this report presents results derived from an investigation of the chemotactic behaviour of ten species of motile bacteria from nine different genera, in which all modes of flagellar arrangement, i.e. monotrichous, lophotrichous and peritrichous, are exhibited. Over I 30 compounds were examined for chemotactic effects. 


\section{METHODS}

Bacteria studied. Alcaligenes faecalis, Bacillus licheniformis, Erwinia carotovora, Escherichia coli K-I2, Proteus morganii, Pseudomonas aeruginosa, Pseudomonas fluorescens, Sarcina ureae, Serratia marcescens and Spirillum serpens were selected from the departmental culture collection as representing a variety of morphological, physiological and flagellar types.

Cultivation and maintenance of bacteria. Stock cultures were grown on slopes of Trypticase Soy Agar (TSA) (Baltimore Biological Laboratories, Baltimore, Maryland, U.S.A.) and thereafter kept at $2 \mathrm{I}$ to $25^{\circ} \mathrm{C}$ with transfers at monthly intervals. Cultures for use in daily experiments were grown in Trypticase Soy Broth (TSB) (Baltimore Biological Laboratories) or on TSA slopes. When the latter was used, growth from the surface of a 14 to I $8 \mathrm{~h}$ culture incubated at $30^{\circ} \mathrm{C}$ was gently washed off with suspending medium (see below). Broth cultures were incubated for I 4 to $\mathrm{I} 8 \mathrm{~h}$ at 27 to $28^{\circ} \mathrm{C}$ in screw-capped vials (I5 $\times$ I $25 \mathrm{~mm}$ ) containing $5 \mathrm{ml}$ TSB on a Temp-Blok incubator (Model 2095, Lab-Line Instruments Inc., Melrose Park, Illinois, U.S.A.).

\section{Chemotaxis experiments}

Culture preparation. Five $\mathrm{ml}$ of a TSA suspension or TSB culture were centrifuged, the pellet resuspended in a buffer (see below) and adjusted to an absorbance of $0.025\left(\sim 3 \times 10^{7}\right.$ colony forming units $/ \mathrm{ml}$ ) at $425 \mathrm{~nm}$.

Flat-glass capillary preparation. Flat-glass capillary tubes (Wright \& Colebrook, I92 I), made from flint glass tubing, having an internal diameter of $8 \mathrm{~mm}$, were cut into $3-\mathrm{cm}$ segments. The final internal cross-sectional dimensions were approximately $0.3 \times \mathrm{I} \cdot 2 \mathrm{~mm}$.

Observations on chemotaxis. The procedure used was that described by Smith \& Doetsch (I969) and Doetsch \& Seymour (1970). Except where indicated, test compounds were incorporated into $1.5 \%(\mathrm{w} / \mathrm{v})$ Ionagar (Oxoid no. 2, Consolidated Laboratories, Chicago, Illinois, U.S.A.), prepared in the buffer used to resuspend the bacteria.

Capillary tubes containing bacterial suspensions were plunged twice into Ionagar, once at the opposite end into Ionagar plus test compound, wiped clean on the exterior with lens paper, mounted on a microscope slide, and examined at 25 to $27^{\circ} \mathrm{C}$ with a Bausch and Lomb 'Stereozoom' variable power microscope (magnification range: 28 to $120 \times$ ). Adjustment of the concave substage mirror provided a 'dark-field' effect. This simple technique permitted observation of individual as well as mass reactions to the developing gradient front within $15 \mathrm{~s}$ of the beginning of an experiment.

Observations were made both at the interface of the 'charged' Ionagar plug and bacterial suspension, and in the region of the advancing gradient front. Negative chemotaxis was recorded when a 'band' or a dense front of bacteria formed and subsequently migrated away from the charged Ionagar plug. This response is illustrated in Smith \& Doetsch (1969). Positive chemotaxis was recorded when bacteria aggregated in a mass or cloud immediately adjacent to the charged Ionagar plug-suspension interface. A positive $(+)$ or negative $(-)$ response was recorded only if after four or more trials with a given species-test compound combination there was at least a $75 \%$ agreement. A variable (v) result was recorded if concurrence was obtained in less than $75 \%$ but more than $40 \%$ of the trials. No response (0) was recorded when no specific effect was observed. Immobilization (i), or cessation of translational movement, was occasionally noted. Individual experiments were of 15 min duration and all reported observations were made during this time. Bacteria immobilized with $5 \%$ $(\mathrm{w} / \mathrm{v})$ formaldehyde, or by heating at $60^{\circ} \mathrm{C}$ for $\mathrm{Io} \mathrm{min}$, and a non-flagellated ( $f a^{-}$) mutant of Pseudomonas fluorescens (Smith \& Doetsch, 1969) served as negative controls. 
Suspending reagents. A phosphate motility buffer similar in composition to those of Adler (I966 b), Smith \& Doetsch (I969) and Doetsch \& Seymour (I970), was used. It contained Io mM-potassium phosphate, I $\mathrm{mM}^{-\mathrm{MgSO}_{4}} \cdot \mathrm{H}_{2} \mathrm{O}$, and $\mathrm{O} \cdot \mathrm{I}$ mM-ethylenediaminetetra-acetic acid (EDTA) at $\mathrm{pH} 77^{\circ}$. When test compounds were added to buffers, bacteria were introduced after their inclusion. When test compounds were incorporated in Ionagar (to make 'charged' Ionagar), they were added to it when the latter was between 45 and $50{ }^{\circ} \mathrm{C}$. When the effects of carbohydrates and amino acids were studied, the phosphate motility buffer

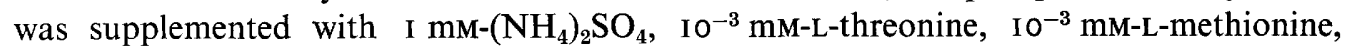
$10^{-3} \mathrm{~mm}-\mathrm{L}-$ leucine, and $10 \mathrm{mM}-\mathrm{D}$-galactose to permit comparison with experiments of Adler (I966a). This buffer was designated as PMBA. Phosphate motility buffer was also fortified with I mM- $\left(\mathrm{NH}_{4}\right)_{2} \mathrm{SO}_{4}$ to make a buffer called PMBN. Suspending solutions were always vigorously mixed with a magnetic stirring device (Mag-Mix, no. 65906, Precision Scientific Co., Chicago, Illinois, U.S.A.) for at least an hour to saturate them with oxygen. This avoided complications due to possible oxygen depletion, or to aerotactic responses. A medium devoid of potassium phosphate, but otherwise the same as phosphate motility buffer, was also used in some experiments to assay the influence of potassium ions, or to obviate solubility problems with some inorganic salts. This was designated as DWMS buffer. All suspending fluids were adjusted to $\mathrm{pH} 7 \cdot 0$ using appropriate reagents.

Compounds suspended in Ionagar. A saturated solution of an inorganic salt was prepared in a given suspending reagent at $\mathrm{pH} 7 \cdot 0$, and then incorporated into molten Ionagar ( $\mathrm{I} \cdot 5 \%$ $\mathrm{w} / \mathrm{v})$ dissolved in the same reagent to give a $10 \%(\mathrm{v} / \mathrm{v})$ amount of this solution in it. Then the agar was allowed to solidify before being used to plug the capillary tubes. Carbohydrates and amino acids of known molecular weight were prepared as $\mathrm{M}$ solutions in $\mathrm{I} \cdot 5 \%(\mathrm{w} / \mathrm{v})$ Ionagar; if the molecular weight was unknown, $\mathrm{I0} \%(\mathrm{w} / \mathrm{v})$ solutions in $\mathrm{I} \cdot 5 \%(\mathrm{w} / \mathrm{v})$ Ionagar were used. Acids and bases were included in Ionagar to give final $\mathrm{pH}$ values as indicated in the text. All compounds used were commercial reagent grades.

\section{RESULTS}

Chemotactic behaviour towards acids and bases. Most of the organic acids surveyed induced negative chemotactic behaviour, and a characteristic 'acidic response band' (Smith \& Doetsch, 1969) developed quickly when bacteria were exposed to them. Similarly, a 'basic response band' developed when the bacteria were exposed to gradients of inorganic bases. The following acids and bases were effective against all bacteria examined: acetic acid at $\mathrm{pH}_{3} \cdot 0$, ascorbic acid at $\mathrm{pH}_{4} \cdot 0$, maleic acid at $3 \cdot 3, \mathrm{HCl}$ at $\mathrm{pH} \mathrm{I} \cdot 0$, and $\mathrm{H}_{2} \mathrm{SO}_{4}$ at $\mathrm{pH} \mathrm{I} \cdot 0 ; \mathrm{NaOH}$ at $\mathrm{pH} \mathrm{I} 2 \cdot 0, \mathrm{KOH}$ at $\mathrm{pH}$ I0.0 to $\mathrm{I} 2 \cdot 0, \mathrm{Ca}(\mathrm{OH})_{2}$ at $\mathrm{pH} \mathrm{I} 2 \cdot 0$, and $\mathrm{NH}_{4} \mathrm{OH}$ at $\mathrm{pH}$ i $1 \cdot 0$. No responses were elicited by EDTA at $\mathrm{pH} 5 \cdot 2$, molybdic acid at $\mathrm{pH} 5 \cdot 4$, mucic acid at $\mathrm{pH} 6 \cdot 5$, or picric acid at $\mathrm{pH} 4 \cdot 3$.

Chemotactic behaviour towards inorganic salts. $\mathrm{Fe}_{2}\left(\mathrm{SO}_{4}\right)_{3}, \mathrm{FeCl}_{3}, \mathrm{Al}_{2}\left(\mathrm{SO}_{4}\right)_{3}$ and $\mathrm{La}\left(\mathrm{NO}_{3}\right)_{3}$, all of which produced final $\mathrm{pH}$ values below $3 \cdot 0$ in Ionagar-phosphate motility buffer plugs, induced negative chemotactic responses in thirty-one out of forty instances (Table $\mathrm{I}$ ). On the other hand, $\mathrm{HgCl}_{2}, \mathrm{NiCl}_{2}$ and $\mathrm{Cu}\left(\mathrm{NO}_{3}\right)_{2}$, whose Ionagar plugs were all above $\mathrm{pH} 3 \cdot 0$, were effective in only six of thirty instances. Curiously, $\mathrm{HgCl}_{2}(\mathrm{pH} 5 \cdot 2$ in Ionagar) failed to induce a negative response from any member of the spectrum tested. Similar results were obtained when the bacteria were suspended in Io mM-tris (hydroxymethyl) aminomethane $\mathrm{HCl}\left(\mathrm{pH}_{7} \cdot 0\right)$ or in distilled water.

Carbohydrates and their analogues as attractants. There was a general lack of chemotactic activity towards the carbohydrates tested (Table 2 ), although nearly every compound served 
Table I. Chemotactic responses to inorganic salts by ten species of motile bacteria

Responses of:

Compound*

None

$\mathrm{Al}_{2}\left(\mathrm{SO}_{4}\right)_{3}$

$\mathrm{CuCl}$

$\mathrm{CuCl}_{2}$

$\mathrm{Cu}\left(\mathrm{NO}_{3}\right)_{2}$

$\mathrm{CuSO}_{4}$

$\mathrm{FeCl}_{3}$

$\mathrm{Fe}_{2}\left(\mathrm{SO}_{4}\right)_{3}$

$\mathrm{HgCl}_{2}$

$\mathrm{La}\left(\mathrm{NO}_{3}\right)_{3}$

$\mathrm{NiCl}_{2}$

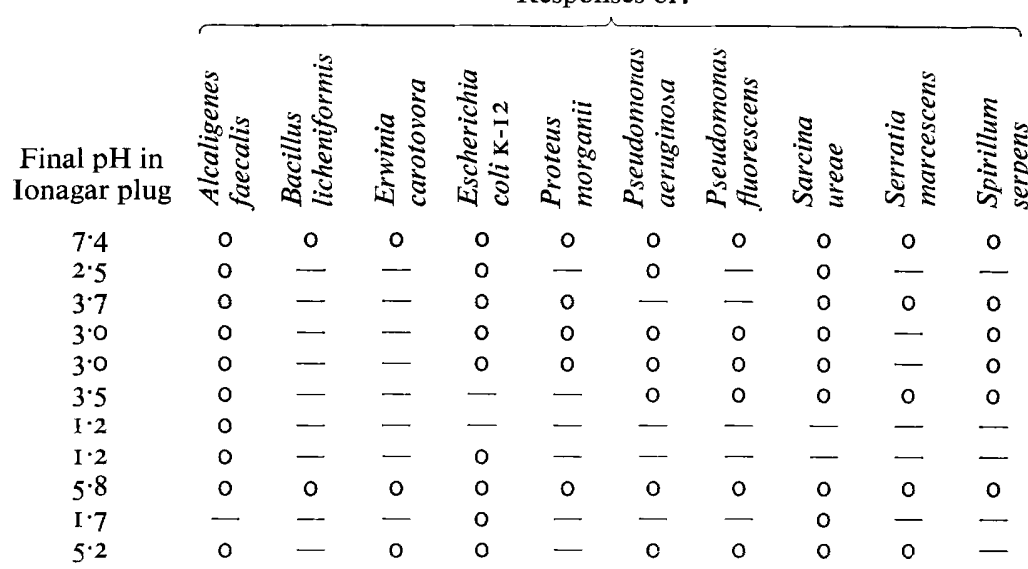

* Incorporated in $\mathrm{I} \cdot 5 \%(\mathrm{w} / \mathrm{v})$ Ionagar as $10 \%(\mathrm{v} / \mathrm{v})$ of saturated solutions in phosphate motility buffer. -, Negative response observed; $\mathrm{o}$, no response observed.

Table 2. Chemotactic responses to carbohydrates by ten species of motile bacteria

Responses of:

\begin{tabular}{|c|c|c|c|c|c|c|c|c|c|c|c|}
\hline $\begin{array}{l}\text { Carbohydrates in } \\
\text { Ionagar plug* }\end{array}$ & $\begin{array}{l}\text { Final } \mathrm{pH} \text { in } \\
\text { Ionagar plug }\end{array}$ & 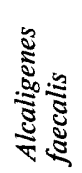 & 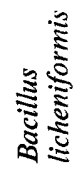 & 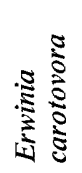 & 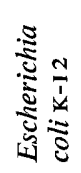 & 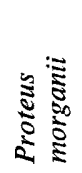 & 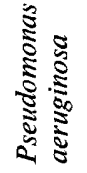 & 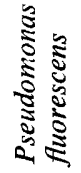 & 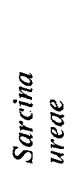 & 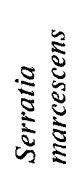 & 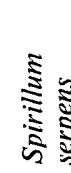 \\
\hline \multicolumn{12}{|l|}{ Monosaccharides } \\
\hline Glycerol & $6 \cdot 6$ & 0 & $+v$ & + & 0 & + & 0 & 0 & 0 & 0 & $+v$ \\
\hline meso-Erythritol & $6 \cdot 3$ & 0 & $+v$ & 0 & 0 & $+v$ & 0 & 0 & 0 & 0 & 0 \\
\hline Adonitol & $6 \cdot 2$ & 0 & 0 & $+v$ & $+v$ & + & + & $+\mathrm{v}$ & 0 & 0 & + \\
\hline D-Arabinose & $6 \cdot I$ & 0 & 0 & + & $+\mathrm{v}$ & 0 & 0 & 0 & 0 & 0 & + \\
\hline L-Arabinose & $6 \cdot I$ & 0 & 0 & $+\mathrm{v}$ & $+v$ & 0 & 0 & 0 & 0 & 0 & + \\
\hline D-Arabitol & $6 \cdot 2$ & 0 & 0 & 0 & 0 & $+\mathrm{v}$ & $+\mathrm{v}$ & $+v$ & 0 & 0 & + \\
\hline L-Arabitol & $6 \cdot 2$ & 0 & o & 0 & 0 & $+v$ & $+\mathrm{v}$ & $+\mathrm{v}$ & 0 & 0 & + \\
\hline D-Lyxose & $6 \cdot 4$ & 0 & o & $+v$ & 0 & + & $+\mathrm{v}$ & + & 0 & 0 & + \\
\hline L-Lyxose & $6 \cdot 3$ & 0 & 0 & + & 0 & + & $+v$ & 0 & 0 & 0 & + \\
\hline D-Ribose & $6 \cdot 0$ & 0 & 0 & 0 & $+\mathrm{v}$ & 0 & $+\mathrm{v}$ & + & 0 & 0 & 0 \\
\hline Xylitol $\dagger$ & $6 \cdot 3$ & 0 & 0 & $+v$ & 0 & 0 & $+\mathrm{v}$ & 0 & 0 & + & + \\
\hline$\alpha$-D-Xylose $\dagger$ & $6 \cdot 3$ & 0 & $+\mathrm{v}$ & + & 0 & $+\mathrm{v}$ & 0 & 0 & 0 & 0 & $+v$ \\
\hline$\beta$-D-Fructose $\dagger$ & $6 \cdot 3$ & 0 & 0 & + & nd & 0 & $+v$ & 0 & 0 & 0 & $+v$ \\
\hline D-Fucose & 6.0 & o & $+v$ & 0 & 0 & $+\mathrm{v}$ & $+\mathrm{v}$ & 0 & 0 & $+v$ & + \\
\hline L-Fucose & $6 \cdot 3$ & 0 & 0 & o & 0 & 0 & 0 & 0 & nd & 0 & 0 \\
\hline Galactitol & 6.8 & 0 & 0 & 0 & 0 & 0 & o & 0 & 0 & 0 & 0 \\
\hline$\alpha-\mathrm{D}-$ Galactose $\dagger$ & $6 \cdot 2$ & 0 & o & + & 0 & 0 & 0 & 0 & 0 & $+v$ & $+\mathrm{v}$ \\
\hline D-Glucose & $6 \cdot 2$ & o & 0 & + & $+v$ & o & 0 & 0 & 0 & $+v$ & $+\mathrm{v}$ \\
\hline D-Glucose $\dagger$ & $6 \cdot 2$ & o & 0 & $+\mathrm{v}$ & nd & 0 & + & 0 & 0 & 0 & 0 \\
\hline 2-Deoxy-D-glucose $\uparrow$ & $6 \cdot 5$ & o & o & nd & 0 & 0 & 0 & 0 & nd & + & 0 \\
\hline iso-Inositol & $6 \cdot 4$ & 0 & 0 & $+\mathrm{v}$ & o & 0 & 0 & o & 0 & 0 & $+v$ \\
\hline D-Mannose & $6 \cdot 2$ & 0 & + & + & $+\mathrm{v}$ & 0 & 0 & 0 & 0 & $+v$ & + \\
\hline D-Mannitol $\dagger$ & $6 \cdot 3$ & 0 & 0 & + & nd & 0 & 0 & 0 & 0 & $+\mathrm{v}$ & + \\
\hline Potassium gluconate & $6 \cdot 6$ & 0 & 0 & $+v$ & $+\mathrm{v}$ & 0 & 0 & 0 & 0 & 0 & $+\mathrm{v}$ \\
\hline L-Rhamnose & $6 \cdot 3$ & 0 & + & + & 0 & + & 0 & 0 & 0 & $+\mathrm{v}$ & + \\
\hline D-Sorbitol & $6 \cdot 3$ & o & 0 & + & $+v$ & $+v$ & $+v$ & + & 0 & 0 & + \\
\hline L-Sorbose & $6 \cdot 6$ & 0 & 0 & + & nd & 0 & 0 & 0 & o & 0 & + \\
\hline Sedoheptulose & $6 \cdot 7$ & 0 & $+v$ & $+v$ & 0 & $+v$ & + & + & 0 & 0 & $+\mathrm{v}$ \\
\hline
\end{tabular}


Table 2 (cont.)

\begin{tabular}{|c|c|c|c|c|c|c|c|c|c|c|c|}
\hline $\begin{array}{l}\text { Carbohydrates in } \\
\text { Ionagar plug* }\end{array}$ & $\begin{array}{l}\text { Final pH in } \\
\text { Ionagar plug }\end{array}$ & 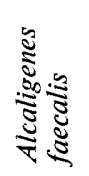 & 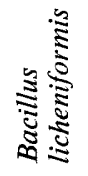 & 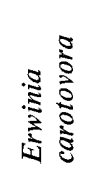 & 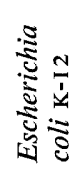 & 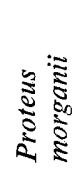 & 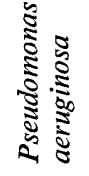 & 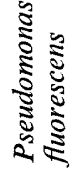 & 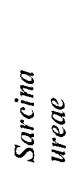 & 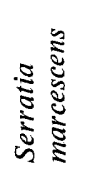 & 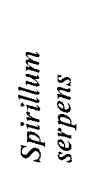 \\
\hline \multicolumn{12}{|l|}{ Disaccharides } \\
\hline D-Cellobiose & $6 \cdot 3$ & o & 0 & o & 0 & 0 & 0 & 0 & 0 & nd & 0 \\
\hline $\begin{array}{l}\text { Cellobiose-octa- } \\
\text { acetate }\end{array}$ & $6 \cdot 6$ & 0 & 0 & 0 & 0 & o & o & 0 & 0 & 0 & 0 \\
\hline D-Lactose. $\mathrm{H}_{2} \mathrm{O}$ & $6 \cdot 4$ & 0 & 0 & + & 0 & 0 & 0 & 0 & 0 & + & $+\mathrm{v}$ \\
\hline Maltose & $6 \cdot 1$ & 0 & o & $+v$ & $+v$ & 0 & o & 0 & nd & o & 0 \\
\hline Melibiose $\dagger$ & $6 \cdot 5$ & 0 & 0 & 0 & 0 & 0 & 0 & 0 & 0 & 0 & o \\
\hline Sucrose ${ }^{\dagger}$ & $6 \cdot 4$ & 0 & 0 & $+\mathrm{v}$ & 0 & 0 & 0 & 0 & 0 & 0 & 0 \\
\hline Trehalose & $6 \cdot 6$ & 0 & 0 & $+v$ & 0 & 0 & 0 & 0 & nd & nd & 0 \\
\hline Turanose & $5 \cdot 2$ & 0 & 0 & 0 & 0 & $+v$ & 0 & o & nd & 0 & $+v$ \\
\hline \multicolumn{12}{|l|}{ Trisaccharides } \\
\hline Melezitose & $6 \cdot 4$ & 0 & 0 & $+v$ & 0 & 0 & $+v$ & o & nd & 0 & 0 \\
\hline Raffinose & 6.6 & o & 0 & 0 & 0 & nd & 0 & 0 & nd & 0 & 0 \\
\hline \multicolumn{12}{|l|}{ Polysaccharides } \\
\hline Arabic acid & $2 \cdot 5$ & - & - & - & - & - & - & - & - & - & - \\
\hline$\beta$-Cyclodextrin & 6.6 & o & 0 & 0 & 0 & 0 & 0 & nd & 0 & 0 & 0 \\
\hline Dextrin & $6 \cdot 3$ & 0 & 0 & 0 & 0 & $+v$ & 0 & $+\mathrm{v}$ & 0 & 0 & o \\
\hline Heparin & $6 \cdot 6$ & 0 & o & 0 & 0 & 0 & 0 & nd & 0 & 0 & nd \\
\hline \multicolumn{12}{|l|}{ Amino sugars } \\
\hline $\mathrm{N}$-Acetyl-glucosamine & 5.6 & 0 & 0 & 0 & 0 & 0 & nd & $+\mathrm{v}$ & nd & 0 & nd \\
\hline $\begin{array}{l}\mathrm{N} \text {-Acetyl- } \\
\text { neuraminic acid }\end{array}$ & $4 \cdot 3$ & o & o & $+v$ & o & nd & 0 & nd & $+v$ & o & 0 \\
\hline \multicolumn{12}{|l|}{ Glycosides } \\
\hline Aesculin & $5 \cdot 8$ & 0 & 0 & 0 & 0 & 0 & 0 & 0 & 0 & $\mathrm{p}$ & nd \\
\hline Aloin & $6 \cdot I$ & 0 & 0 & 0 & 0 & 0 & nd & nd & o & 0 & nd \\
\hline Arbutin & $6 \cdot 2$ & 0 & o & 0 & 0 & nd & 0 & + & 0 & 0 & nd \\
\hline $\begin{array}{l}\alpha \text {-Methyl-D- } \\
\text { glucoside } \dagger\end{array}$ & $6 \cdot 3$ & 0 & 0 & + & nd & 0 & 0 & 0 & 0 & + & 0 \\
\hline $\begin{array}{l}\alpha \text {-Methyl-D- } \\
\text { mannoside } \dagger\end{array}$ & $5 \cdot 5$ & 0 & 0 & + & 0 & 0 & 0 & + & 0 & 0 & \\
\hline Salicin & $6 \cdot 4$ & 0 & 0 & 0 & $+\mathrm{v}$ & 0 & 0 & 0 & 0 & 0 & o \\
\hline
\end{tabular}

* Arranged alphabetically in order of increasing number of carbon atoms in the basic carbohydrate unit.

$\uparrow$ PMBN used instead of PMBA.

+ , Positive response; -, negative response; o, no response; v, variable response; nd, not determined. M concentrations were used except for: (I) aloin, dextrin and heparin, which were incorporated in $I .5 \%$ $(\mathrm{w} / \mathrm{v})$ Ionagar as $10 \%$ solutions; and (2) aesculin, arbutin, cellobiose, cellobiose octa-acetate, $\beta$-cyclodextrin and raffinose, which were incorporated in $1.5 \%(\mathrm{w} / \mathrm{v})$ Ionagar in $10 \%(\mathrm{v} / \mathrm{v})$ amounts of saturated solutions. PMBA was used to suspend bacteria and as solvent for the carbohydrates.

as an attractant for at least one species and only one compound, arabic acid (galactoaraban), evoked a uniformly negative chemotactic response. Only Alcaligenes faecalis of the bacteria tested, failed to respond to any compound. When PMBA buffer, which contains D-galactose, was used, a response to D-galactose was not expected. However, responses to its analogues, D-fucose (6-deoxy-D-galactose) and galactitol, to compounds with homologous skeletal segments, L-arabinose, D-lyxose, L-sorbose, L-sorbitol and D-xylose, and to D-galactosecontaining compounds, D-lactose, melibiose, and raffinose, were possible. In fact, many of these were found to be attractants. In the cases of arabinose, arabitol and lyxose, both Dand L-forms were attractants for a given organism.

There was no evidence of a 'universal' attractant. The highest frequency of attraction (6 of Io species) was to adonitol (ribitol), sedoheptulose and D-sorbitol. The first two share 
a ribose moiety consisting of four $-\mathrm{CH}_{2} \mathrm{OH}$ groups, whilst D-sorbitol has a homologous configurational structure at $\mathrm{C} 4,5,6$. Pseudomonas fluorescens and Pseudomonas aeruginosa had a few attractants in common, but each responded to some compounds that the other did not. The most responsive organism was Erwinia carotovora, which was attracted to twenty-six different compounds. A greater number of monosaccharides were attractants than any other class of carbohydrates examined.

When PMBN, which does not contain D-galactose, L-methionine, L-threonine or L-leucine, was used in place of PMBA, responses to D-galactose, its analogues, compounds with identical skeletal moieties and compounds containing D-galactose, could be examined without possible interference. In the case of $\alpha$-D-xylose, PMBA appeared to block a positive response by Proteus morganii, but had no effect on that of Erwinia carotovora. With D-fucose, there was an indication of interference by PMBA with the positive chemotactic response recorded for Serratia marcescens; likewise, the response by Erwinia carotovora towards D-galactose appeared to be blocked by PMBA. Adler (I969) reported that the presence of a galactose chemoreceptor may be accompanied by a reduced response towards D-glucose, $\alpha$-methyl-D-glucoside and 2-deoxyglucose. Similar results were obtained with Serratia marcescens, which showed positive chemotaxis towards 2-deoxyglucose and $\alpha$-methyl-Dglucoside, as well as to D-galactose, but not towards D-glucose unless PMBA was the solvent.

If D-glucose served as an attractant for a particular species of bacteria, several analogues, compounds containing homologous skeletal moieties and a few glucose-containing compounds also evoked positive responses. Similarly, D-mannose, its structural analogue, L-rhamnose, and D-lyxose, which possesses a homologous skeletal moiety to C I, 2, 3, 4 of D-mannose, served equally as attractants for Erwinia carotovora and Spirillum serpens. Although containing glucose units, disaccharides or more complex carbohydrate molecules rarely evoked positive chemotactic responses.

There were correlations in positive responses to adonitol and compounds sharing homologous $\mathrm{C}_{1}, 2,3$ moieties, i.e. D- and L-arabinose $\left(\mathrm{C}_{3}, 4,5\right)$, D- and L-arabitol $\left(\mathrm{C}_{3}, 4,5\right)$, meso-erythritol (C I, 2, 3), D-glucose $\left(\mathrm{C}_{4}, 5,6\right)$, D-mannose $\left(\mathrm{C}_{4}, 5,6\right)$, D-mannitol $\left(\mathrm{C} \mathrm{I}_{1}, 2,3\right)$ and D-sorbitol $\left(\mathrm{C}_{4}, 5,6\right)$. Compounds sharing homologous skeletal moieties at $\mathrm{C} \mathrm{I,} \mathrm{2,} 3$ of D-arabitol, other than D-galactose $\left(\mathrm{C}_{4}, 5,6\right)$, i.e. D-lyxose $\left(\mathrm{C}_{3}, 4,5\right)$, xylitol $\left(\mathrm{C}_{1}, 2,3\right)$, and D-xylose $\left(\mathrm{C}_{3}, 4,5\right)$, did not, however, attract any organism of the spectrum examined.

Chemotactic effects of amino acids. Responses of bacteria to amino acids at about $\mathrm{pH} 7 \cdot 0$ were more variable than towards carbohydrates, but each species responded uniquely (Table 3). In some instances, especially with L-cysteine, test bacteria were immobilized on contact with the gradient front. Escherichia coli was neither attracted nor repelled by any amino acid, regardless of whether the system consisted of PMBA or PMBN. With Proteus morganii, when PMBA was used there was a positive chemotactic response to L-histidine and DL-isoleucine, whereas there was no response in PMBN. There were also instances wherein positive responses were observed in PMBN but not PMBA, for example: L-arginine for Proteus morganii; glycine, L-histidine, DL-isoleucine and L-proline for Bacillus licheniformis; and L-histidine for Spirillum serpens.

Effects of gramicidin D on chemotaxis. Gramicidin D is known to have complex effects on membranes, and since flagellar orientation may be controlled to some degree by membrane-mediated events (Faust \& Doetsch, 197 I Doetsch, I972), chemotactic responses were of interest. In most instances, various formulations containing gramidicin D did not affect the motile behaviour of Pseudomonas fluorescens (Table 4). However, in all cases except gramicidin D in distilled water, a negative response similar to that developed toward acid gradients was observed. 
Table 3. Chemotactic responses to amino acids by ten species of motile bacteria

Responses of:

Amino acid in Ionagar plug*

DL- $\alpha$-Alanine L-Arginine

L-Aspartic acid

L-Cysteine $\mathrm{HCl}$

L-Cystine

L-Sodium glutamate

Glycine

L-Histidine

L-Hydroxyproline

DL-Isoleucine

L-Leucine

L-Lysine $\mathrm{HCl}$

L-Methionine

L-Phenylalanine

L-Proline

DL-Serine

L-Threonine

L-Tryptophan

L-Tyrosine

DL-Valine

\begin{tabular}{|c|c|c|c|c|c|c|c|c|c|c|}
\hline \multirow[b]{2}{*}{$\begin{array}{l}\text { Final pH of } \\
\text { Ionagar plug }\end{array}$} & \multicolumn{10}{|c|}{ Responses of: } \\
\hline & 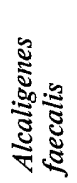 & 莺 & 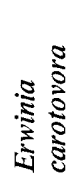 & 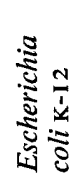 & 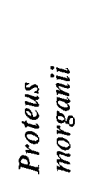 & 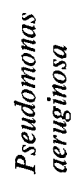 & 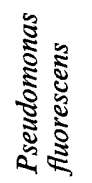 & 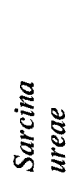 & 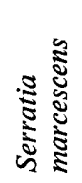 & 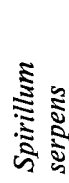 \\
\hline $7 \cdot 0$ & 0 & $+v$ & $+\mathrm{i}$ & 0 & + & $+\mathrm{v}$ & $+v$ & 0 & 0 & + \\
\hline $7 \cdot 6$ & $-v$ & - & $\mathrm{i}$ & $\mathrm{i}$ & $+v$ & $-\mathrm{v}$ & $-v$ & - & 0 & - \\
\hline $6 \cdot 7$ & - & 0 & $\mathrm{i}$ & 0 & $+\mathrm{v}$ & $+v$ & - & $+\mathrm{v}$ & 0 & $+v$ \\
\hline $7 \cdot 0$ & - & $+\mathrm{i}$ & $\mathrm{i}$ & 0 & $+\mathrm{i}$ & $\mathrm{i}$ & $+v$ & $\mathrm{i}$ & $\mathrm{i}$ & $\mathrm{i}$ \\
\hline $6 \cdot 9$ & 0 & 0 & 0 & 0 & 0 & $-\mathrm{v}$ & $-v$ & 0 & o & o \\
\hline $6 \cdot 0$ & 0 & $+v$ & $\mathrm{i}$ & 0 & 0 & 0 & $-v$ & 0 & 0 & 0 \\
\hline $7 \cdot 0$ & 0 & $+v$ & $\mathrm{i}$ & 0 & $+v$ & 0 & 0 & 0 & $+v$ & + \\
\hline $7 \cdot 5$ & 0 & + & 0 & 0 & 0 & 0 & 0 & 0 & 0 & $+\mathrm{v}$ \\
\hline $7 \cdot 0$ & 0 & 0 & 0 & 0 & $+v$ & 0 & 0 & 0 & 0 & $+v$ \\
\hline $7 \cdot 0$ & 0 & + & 0 & 0 & 0 & $+v$ & $+v$ & 0 & 0 & $+v$ \\
\hline $6 \cdot 9$ & 0 & + & 0 & 0 & + & $+\mathrm{v}$ & 0 & $\mathrm{i}$ & 0 & 0 \\
\hline $6 \cdot 8$ & - & - & - & 0 & - & - & - & - & - & - \\
\hline $6 \cdot 8$ & 0 & + & 0 & 0 & + & $+v$ & + & $\mathrm{i}$ & $+v$ & 0 \\
\hline $7 \cdot 0$ & 0 & $+v$ & 0 & 0 & + & 0 & $+v$ & $\mathrm{i}$ & 0 & 0 \\
\hline $6 \cdot 7$ & 0 & + & 0 & 0 & $+v$ & 0 & 0 & 0 & 0 & $+v$ \\
\hline $6 \cdot 9$ & 0 & + & 0 & 0 & + & 0 & + & 0 & 0 & 0 \\
\hline $6 \cdot 7$ & 0 & $+v$ & 0 & 0 & + & 0 & + & 0 & 0 & $+v$ \\
\hline $6 \cdot 7$ & 0 & + & 0 & 0 & + & 0 & 0 & 0 & $+v$ & 0 \\
\hline $6 \cdot 4$ & 0 & 0 & 0 & 0 & 0 & 0 & 0 & 0 & 0 & 0 \\
\hline $6 \cdot 7$ & 0 & 0 & 0 & 0 & + & 0 & + & $\mathrm{i}$ & 0 & $+\mathrm{v}$ \\
\hline
\end{tabular}

L-tyrosine, which were incorporated in $1.5 \%(\mathrm{w} / \mathrm{V})$ Ionagar as saturated solutions.

+ , Positive chemotaxis; - , negative chemotaxis; o, no chemotactic response; $i$, immobilized; $v$, variable response.

Effects of lipid solvents on negative chemotactic behaviour. Certain solvents provoked an increase in the number of reversals in translational movement, thereby markedly decreasing the average mean free paths of bacteria suspended in them. These included $(\% \mathrm{v} / \mathrm{v}):$ carbontetrachloride (0.00I), chloroform (0.0I), hexane (0.0I), acetone (I) and propanol (I). Many compounds uncoupled the response of Pseudomonas fluorescens to acid or base gradients ( $\mathrm{pH} \mathrm{I}^{\circ} \mathrm{O}$ and $\mathrm{I} 2.0$ respectively) from translational movement, without greatly interfering with it. Included among these compounds $(\% \mathrm{v} / \mathrm{v})$ were: benzene $(0.08)$, carbon tetrachloride $(0.01)$, acetone (I), diethyl ether (0.0I), hexane (0.0I), carbon disulphide $(0.2)$, chlorobenzene $(0.04)$, chloroform (0.0I) and propanol(I). At the concentrations employed, benzene, hexane, carbon disulphide and chlorobenzene were saturated solutions. Toluene, ethanol and butanol did not uncouple these responses at any concentration level employed, but gradients of aliphatic alcohols, in general, induced a negative chemotactic response.

Miscellaneous effects. Chloral hydrate (I $\mathrm{mM}$ ) when included in phosphate motility buffer-Ionagar, prevented a negative chemotactic response by Pseudomonas fluorescens to an acid ( $\left.\mathrm{pH} \mathrm{r}^{\circ} \mathrm{O}\right)$ gradient, without noticeably affecting translational movement.

Adenosine-3',5'-cyclic monophosphate (M neutralized to $\mathrm{pH} 6.8$ in PMBN-Ionagar) induced a positive chemotactic response only in Pseudomonas fluorescens and Pseudomonas aeruginosa among the bacteria of the test spectrum. 
Table 4. Response of Pseudomonas fluorescens to various formulations incorporating gramicidin $D$

\begin{tabular}{|c|c|c|c|c|c|}
\hline \multirow[b]{2}{*}{$\begin{array}{l}\text { Chemicals in } \\
\text { Ionagar* }\end{array}$} & \multirow[b]{2}{*}{$\begin{array}{l}\text { Final } \mathrm{pH} \text { in } \\
\text { Ionagar plug }\end{array}$} & \multirow[b]{2}{*}{$\begin{array}{c}\text { Composition of } \\
\text { bacterial suspension } \\
\text { medium }\end{array}$} & \multicolumn{3}{|c|}{ Observations } \\
\hline & & & Motility & $\begin{array}{l}\text { Response band } \\
\text { formed? }\end{array}$ & $\begin{array}{l}\text { Behaviour in } \\
\text { response band }\end{array}$ \\
\hline $\mathrm{HCl}, \mathrm{PMB}$ & $I \cdot 0$ & PMB & Normal & Yes & Normal \\
\hline GMCD, PMB & $6 \cdot 3$ & PMB & Normal & Yes & Normal \\
\hline $\mathrm{HCl}, \mathrm{DW}$ & $\mathrm{I} \cdot \mathrm{O}$ & DW & Slow & Yes & Speed increased \\
\hline GMCD, DW & $6 \cdot 0$ & DW & Slow & No & Variable \\
\hline GMCD, PMB & $6 \cdot 3$ & $\mathrm{DW}$ & Slow & Yes & Speed increased \\
\hline GMCD, DW & $6 \cdot 0$ & PMB & Normal & Yes & Normal \\
\hline $\mathrm{HCl}, \mathrm{DW}$ & I.O & ${ }^{10^{-2}} \mathrm{M} \mathrm{NH}_{4} \mathrm{Cl}, \mathrm{DW}$ & Normal & Yes & Normal \\
\hline GMCD, DW & $6 \cdot 0$ & $\mathrm{IO}^{-2} \mathrm{M} \mathrm{NH}_{4} \mathrm{Cl}, \mathrm{DW}$ & Normal & Yes & Normal \\
\hline $\mathrm{HCl}, \mathrm{DW}$ & $I \cdot 0$ & $\mathrm{IO}^{-2} \mathrm{M} \mathrm{NaCl}, \mathrm{DW}$ & Slow & Yes & Normal \\
\hline GMCD, DW & $6 \cdot 0$ & $\mathrm{I}^{-2} \mathrm{M} \mathrm{NaCl}, \mathrm{DW}$ & Slow & Yes & Normal \\
\hline
\end{tabular}

* $\mathrm{HCl}$ was added to give $\mathrm{pH} \mathrm{I} \cdot \mathrm{O}$ in Ionagar.

PMB, Phosphate motility buffer; DW, distilled water; GMCD, gramicidin as a saturated solution.

\section{DISCUSSION}

Molecules or ions (diffusing from Ionagar plugs) that were likely to exert harmful or lethal effects, evoked negative chemotactic responses. Thus, organic and inorganic acids, inorganic bases, and inorganic salts, whose solutions were acidic $\left(\mathrm{pH} \mathrm{I}^{\circ} \mathrm{O}\right.$ to $\left.3 \cdot 0\right)$ or basic $(\mathrm{pH} 10 \cdot 0$ to $12 \cdot 0)$, induced the development of a migrating band of bacteria whose direction was away from the advancing gradient front. There appears to be survival value in the ability of a bacterium to 'sense' its environment in these instances and move away from physiologically inimical (low and high $\mathrm{pH}$ ) areas. It seems as unlikely that bacteria would possess specific 'metal chemoreceptors' for $\mathrm{Al}^{3+}, \mathrm{La}^{3+}, \mathrm{Fe}^{3+}, \mathrm{Cu}^{2+}$ or $\mathrm{Ni}^{2+}$, as it is to expect them to have $\mathrm{Cl}^{-}, \mathrm{NO}_{3}^{-}$, acetate or ascorbate receptors. A simpler explanation is that those inorganic salts, whose ionization in solutions results in low or high $\mathrm{pH}$ values, induce negative responses in sensitive organisms by acting as fairly strong acids or bases. Experiments in which the charged Ionagar plugs were neutralized to about $\mathrm{pH} 7 \cdot 0$, or where the strength of the buffer in which the bacteria were suspended was increased, resulted in abolition of, or marked inhibition of, band formation. If the bacteria were suspended directly into solutions of acids, bases or salts used for charging Ionagar plugs, they were uniformly rendered non-motile and frequently killed. The general capacity of bacteria to respond to low and high $\mathrm{pH}$ conditions suggests that some common mechanism is involved, and a theory attempting to unify all motile behaviour was recently proposed (Doetsch, 1972).

Some toxic compounds are not sensed by bacteria: no bacterium examined moved away from $\mathrm{HgCl}_{2}$ and results obtained with gramicidin $\mathrm{D}$ suggest that a compound need not necessarily be strongly acidic or basic to evoke a negative chemotactic response. Alteration of membrane potentials (Faust \& Doetsch, 197I; Doetsch, 1972) due to changes of cation permeability may be one common feature of all tactic responses coupled directly to flagellar reorientation, and hence, translational movement. The response to gramicidin $\mathrm{D}$ might be explained in this manner. There was not, however, a single instance wherein any organism responded positively to a potentially lethal or toxic agent.

Results obtained from studies on the effects of carbohydrates and amino acids on the spectrum of bacteria apparently allow few generalizations. There is no explanation as to why Alcaligenes faecalis failed to show a positive chemotactic response to any carbohydrate 
or amino acid. The lack of response of Erwinia carotovora and Escherichia coli $\mathrm{K}-\mathrm{I} 2$ to any amino acid is also puzzling, particularly as Erwinia carotovora responded to more than twenty carbohydrates.

Positive chemotaxis towards amino acids was only infrequently observed, but when a given species showed this behaviour, it did so for many of them, as, for example, did Bacillus licheniformis and Proteus morganii. Mesibov \& Adler (1972) reported that Escherichia coli was attracted, under their experimental conditions, to alanine, aspartate, cysteine, glutamic, glycine, methionine, serine, and threonine. They concluded that this organism probably had two detection systems, namely, aspartate and serine 'chemoreceptors'. Armstrong (I972) pointed out that methionine auxotrophs of Escherichia coli required methionine in the medium in order to exhibit chemotactic responses.

Effects of lipid solvents in uncoupling chemotactic responses from normal translational movements are worthy of further study. Fogel \& Mitchell (I97I), and Chet, Fogel \& Mitchell (197I), reported that positive responses of a marine Pseudomonas species were uncoupled in the presence of $0.5 \%(\mathrm{v} / \mathrm{v})$ ethanol. Ethanol could 'mask' possible receptors without irreversibly damaging them, since positive responses were re-initiated upon washing the organisms free of ethanol. The observations made here suggest that membrane-associated elements are reversibly altered by benzene, diethyl ether, acetone, chloroform and other solvents, possibly by changes in dielectric constant of the medium.

The demonstration of a positive chemotactic response toward a given compound by a given bacterium is probably directly dependent upon a number of laboratory-dictated conditions (Adler, I973). Whether these requirements are often encountered in natural environments is a moot question. Medium composition, temperature of incubation and testing, composition of suspending fluids, substrate concentration and the like, apparently do have a great influence on the experimental results of laboratory-observed chemotaxis. Furthermore, Adler (I969) has shown that Escherichia coli is attracted to compounds it cannot metabolize or to ones it cannot transport and, in fact, some key metabolites do not even serve as attractants for these organisms. Indeed, many of the compounds acting as attractants for specific organisms reported in this present work are neither fermented, oxidized, or used as sole carbon or nitrogen sources by them.

Our work suggests that whereas positive chemotactic responses may be only of fortuitous and occasional value to bacteria under natural environmental conditions, negative chemotactic responses nearly always develop toward lethal or hostile chemical gradients. These are probably not spurious or redundant responses since they have definite survival value. Whilst bacteria may be equipped with a complement of receptors to detect specific compounds toward which they may thereby be attracted, it seems unnecessary to postulate specific receptors to explain behaviour toward the variety of toxic compounds from which they move away (Doetsch \& Seymour, 1970; Doetsch, 1972), and there is probably a distinct difference in the mechanisms of positive and negative chemotaxis.

This work was supported in part by a grant from the U.S. Department of Health, Education and Welfare, USPHS, National Institute of Allergy and Infectious Diseases, Grant AI-0926I-03. 


\section{REFERENCES}

AdLer, J. (1966a). Chemotaxis in bacteria. Science, New York 153, 708-716.

ADLER, J. (1966 b). Effect of amino acids and oxygen on chemotaxis in Escherichia coli. Journal of Bacteriology 92, I 2 I-I 29.

Adler, J. (1969). Chemoreceptors in bacteria. Science, New York 166, 1588-1597.

ADLER, J. (I973). A method for measuring chemotaxis and use of the method to determine optimum conditions for chemotaxis in Escherichia coli. Journal of General Microbiology 74, 77-91.

Armstrong, J. B. (1972). Chemotaxis and methionine metabolism in Escherichia coli. Canadian Journal of Microbiology 18, 59I-596.

Berg, H. C. \& Brown, D. A. (1972). Chemotaxis in Escherichia coli analysed by three-dimensional tracking. Nature, London 239, 500-504.

Chet, I., Fogel, S. \& Mitchell, R. (197I). Chemical detection of microbial prey by bacterial predators. Journal of Bacteriology ro6, 863-867.

Clayton, R. K. (1958). On the interplay of environmental factors affecting taxis and motility in Rhodospirillum rubrum. Archiv für Mikrobiologie 29, I89-2 I 2.

Clayton, R. K. (1964). Phototaxis in micro-organisms. In Photophysiology, vol. 2. Edited by A. C. Giese. New York: Academic Press.

Dahlquist, F. W., Lovely, P. \& Koshland, D. E., JUN. (1972). Quantitative analysis of bacterial migration in chemotaxis. Nature New Biology, London 236, $120-123$.

Doetsch, R. N. (197r). Functional aspects of bacterial flagellar motility. Critical Reviews in Microbiology I, 73-103.

Doetsch, R. N. (1972). A unified theory of bacterial motile behaviour. Journal of Theoretical Biology 35 , $55-66$.

DoETSCH, R. N. \& HAGEAGE, G. J. (I968). Motility in procaryotic organisms: problems, points of view, and perspectives. Biological Reviews of the Cambridge Philosophical Society 43, 317-362.

Doetsch, R. N. \& Seymour, F. W. K. (1970). Negative chemotaxis in bacteria. Life Sciences 9, 1029-1037.

ECKERT, R. (1972). Bioelectric control of ciliary activity. Science, New York $\mathbf{1 7 6}, 473-48$ I.

FAUST, M. A. \& DoETSCH, R. N. (I97I). Effect of membrane-active antibiotics on motility and ${ }^{42} \mathrm{~K}$ permeability of Pseudomonas fuorescens. Canadian Journal of Microbiology 17, 183-189.

Fogel, S. \& Mrtchell, R. (I97I). The ecological significance of bacterial chemotaxis. Bacteriological Proceedings, 28.

GAMOw, R. I., BotTGER, B. \& BARNES, F. S. (I97I). Analysis of chemotaxis in white blood cells. Biophysical Journal II, 860-867.

Hazelbauer, G. L. \& Adler, J. (197I). Role of the galactose binding protein in chemotaxis of Escherichia coli toward galactose. Nature New Biology, London 230, IOI-IO4.

KalCKAR, H. M. (1971). The periplasmic galactose binding protein of Escherichia coli. Science, New York I74, 557-565.

Konijn, T. M., van de Meene, J. G. J., Chang, Y. Y., Barkley, D. S. \& Bonner, J. T. (I969). Identification of adenosine- $3^{\prime}, 5^{\prime}$-monophosphate as the bacterial attractant for myxamoebae of Dictyostelium discoideum. Journal of Bacteriology 99, 510-51 2.

Macnab, R. M. \& Koshland, D. E., Jun. (1972). The gradient-sensing mechanism in bacterial chemotaxis. Proceedings of the National Academy of Sciences of the United States of America 69, 2509-25I 2.

Mesibov, R. \& AdLer, J. (1972). Chemotaxis toward amino acids in Escherichia coli. Journal of Bacteriology II2, 3I 5-326.

NAITOH, Y. \& ECKERT, R. (I969a). Ionic mechanisms controlling behavioral responses of Paramecium to mechanical stimulation. Science, New York r64, 963-965.

NAITOH, Y. \& ECKERT, R. (1969b). Ciliary orientation: controlled by cell membrane or by intracellular fibrils? Science, New York 166, 1633-1635.

SMIth, J. \& Doetsch, R. N. (I969). Studies on negative chemotaxis and the survival value of motility in Pseumomonas fuorescens. Journal of General Microbiology 55, 379-39I .

Wright, A. E. \& Colebrook, L.(192I). Technique of the Teat and Capillary Tube, p. 22. London: Constable. 